\title{
Constraining the Properties of the Interstellar Turbulence around Geminga using HAWC Measurements
}

\author{
Gwenael Giacinti* \\ Max-Planck-Institut für Kernphysik, Postfach 103980, 69029 Heidelberg, Germany \\ E-mail: giacinti@mpi-hd.mpg.de
}

Rubén López-Coto

Università di Padova and INFN, I-35131, Padova, Italy

E-mail: rlopezepd.infn.it

\begin{abstract}
Extended gamma-ray emissions around sources of cosmic-rays can be used as probes of the interstellar magnetic fields around these sources. Using HAWC measurements in the Geminga region, we put constraints on the parameters of the turbulence within $\simeq 25 \mathrm{pc}$ from Geminga pulsar. We propagate $\sim 100 \mathrm{TeV}$ electrons in 3D turbulence, and compare their gamma-ray emission with the measurements. We find that the measurements are compatible with expectations for Kolmogorov or Kraichnan turbulence with reasonable root-mean-square strengths $B_{\mathrm{rms}} \approx 3 \mu \mathrm{G}$ and coherence lengths $l_{\mathrm{c}} \approx 1 \mathrm{pc}$.
\end{abstract}

36th International Cosmic Ray Conference -ICRC2019-

July 24th - August 1st, 2019

Madison, WI, U.S.A.

\footnotetext{
* Speaker.
} 


\section{Introduction}

Extended gamma-ray emissions around Galactic sources of cosmic-rays (CR) can provide insights into the properties of the turbulent magnetic fields that surround these sources, see References $[1,2]$. Such an emission has been detected by the HAWC Observatory around Geminga, and is thought to be due to $\approx 100 \mathrm{TeV}$ electrons and positrons diffusing and cooling around the pulsar [3]. The HAWC Collaboration measured the value of the diffusion coefficient of these electrons, and found it to be $\mathscr{D}_{100}=(4.5 \pm 1.2) \times 10^{27} \mathrm{~cm}^{2} \mathrm{~s}^{-1}$ at $100 \mathrm{TeV}$. Its extrapolation to lower energies is about two orders of magnitude smaller than the "Galactic average" as deduced from the boron-to-carbon ratio. In the present work, we place constraints on the turbulence within $\approx 25 \mathrm{pc}$ from Geminga, using the measurements published in [3]. We find that 3D isotropic Kolmogorov or Kraichnan turbulence with a coherence length $l_{\mathrm{c}} \approx 1 \mathrm{pc}$ provides a good fit to the measurements. See also Reference [4] for more details.

\section{Numerical simulations}

We describe here how we produce the synthetic gamma-ray maps that are compared with HAWC measurements in the next section. Instead of using the diffusion-loss equation, we propagate individual very-high-energy electrons in realizations of 3D isotropic Kolmogorov or Kraichan turbulence. We use 5000 particles for each map. This technique allows us to take into account effects that cannot be described properly within the standard isotropic diffusion approximation, such as highly anisotropic propagation of CRs along magnetic field lines: When the particles that escaped from a source are still located at distances smaller than $\approx l_{\mathrm{c}}$ from it, their gamma-ray emission is expected to highlight magnetic field lines around the source and, therefore, appear filamentary.

The nature of these particles, i.e. electrons and/or positrons, is not important for the conclusions of the present study. We note that one often assumes that pulsars accelerate electrons and positrons in equal quantities. However, this may not be the case at the highest energies: We have discovered in Ref. [5] that pulsars can favour one type of particles (electrons or positrons, but not both) at the highest energies, if particle acceleration takes place in the equatorial region of the pulsar wind termination shock.

Measurements of the gamma-ray spectrum of Geminga in Reference [3] shows that it follows a power law $d N_{\gamma} / d E \propto E^{-2.34}$ between $8 \mathrm{TeV}$ and $40 \mathrm{TeV}$. This is compatible with electrons being injected with a spectrum $d N_{\mathrm{e}} / d E \propto E^{-2.24}$ at the pulsar. In the simulations, we inject the electrons at the location of the source, which we assume to be point-like. The initial energies of these electrons are chosen between $40 \mathrm{TeV}$ and $500 \mathrm{TeV}$ and follow a power-law spectrum $\propto E^{-2.24}$. We take into account their synchrotron and inverse Compton energy losses during propagation, which are the dominant losses at the energies we consider, see Reference [6]. The effect of infrared and optical photons is neglected. The energy loss per time unit of an electron with energy $E$ and placed in a magnetic field $B$ reads [7]:

$$
\left|\frac{d E}{d t}\right| \simeq 2.53 \times 10^{-15} \mathrm{TeV} / \mathrm{s}\left[\left(\frac{B}{1 \mu \mathrm{G}}\right)^{2}+\frac{10.1}{(1+E /(99 \mathrm{TeV}))^{1.5}}\right]\left(\frac{E}{1 \mathrm{TeV}}\right)^{2} .
$$


We generate the 3D turbulent magnetic fields in which we propagate these particles with the method presented in Reference [8]. We test both isotropic Kolmogorov $\left(\mathscr{P}(k) \propto k^{-5 / 3}\right)$ and Kraichnan $\left(\mathscr{P}(k) \propto k^{-3 / 2}\right)$ turbulence with root-mean-square strengths in the range $B_{\mathrm{rms}}=2-5 \mu \mathrm{G}$ and coherence lengths in the range $l_{\mathrm{c}}=0.1-40 \mathrm{pc}$. Trajectories are stopped once the particles reach $39 \mathrm{TeV}$, because lower energy electrons do not contribute to the range of photon energies considered here. Our turbulence contains fluctuations down to scales smaller than the smallest electron gyroradius present in our simulations, so as to ensure particle scattering. Since HAWC measurements do not show any strong asymmetry in the emission, we do not add any large-scale magnetic field to our turbulence, see the discussion in Section 4. Since the typical cooling time of $100 \mathrm{TeV}$ electrons is only $\sim 10 \mathrm{kyr}$, we assume that the electrons have been injected steadily on these time scales. Instead of injecting the electrons continuously in the simulation, we inject them at $t=0$, and record their momenta and positions at equally spaced intervals in time, every $\Delta t$, and consider each recording as a new particle for the total emission. We verified that $\Delta t=20 \mathrm{yr}$ gives correct results. The gamma-ray emission is dominated here by the upscattering of CMB photons. We calculate it, using the gamera [9] and edge [6] libraries. Finally, we normalize our total gamma-ray emission to that measured by HAWC - the number of electrons in our simulations being much smaller than that present around Geminga in reality.

\section{Results}

In Figure 1, we plot the simulated gamma-ray surface brightness in a $10^{\circ}$-radius region around Geminga, when viewed from the Earth. The pulsar is in the centre of each plot. We use here 3D isotropic Kolmogorov turbulence with root-mean-square strength $B_{\mathrm{rms}}=3 \mu \mathrm{G}$. In each panel, the coherence length is set to a different value: $l_{\mathrm{c}}=0.25 \mathrm{pc}$ (in the top left panel), $1 \mathrm{pc}$ (top right), $5 \mathrm{pc}$ (middle left), $10 \mathrm{pc}$ (middle right), $20 \mathrm{pc}$ (bottom left), and $40 \mathrm{pc}$ (bottom right). The brightest regions are in red and yellow. Cf. the colourbars on the side of the panels for the surface brightness values. The emission clearly becomes increasingly asymmetric with respect to rotations around the position of the pulsar for increasing values of $l_{\mathrm{c}}$. This is due to the fact that neighbouring magnetic field lines close to the source remain close to one another typically up to distances $\approx l_{\mathrm{c}}$ from the source. For small values of the coherence length, $l_{\mathrm{c}} \lesssim 5 \mathrm{pc}$, magnetic field lines are tangled on scales that are significantly smaller than the size of the gamma-ray emitting region. Hence, even if CRs follow magnetic field lines individually, their gamma-ray emission around the source looks rather symmetric to the observer. In contrast, for larger values of the coherence length, $l_{\mathrm{c}} \gtrsim 10 \mathrm{pc}$, it is not small any more compared to the size of the gamma-ray emitting region. In this case, the bulk of escaping CRs is mostly confined in a magnetic flux tube of length $\lesssim l_{\mathrm{c}}$ around the source. The filamentary structures visible in gamma-rays follow the local magnetic field lines. The presence or absence of asymmetries in the emission can then be used to put constraints on $l_{c}$. Since no strong asymmetry has been detected by HAWC yet, the results in Figure 1 allow us to set the upper limit $l_{\mathrm{c}} \lesssim 5 \mathrm{pc}$. The simulated emissions for $l_{\mathrm{c}} \gtrsim 10 \mathrm{pc}$ are clearly too asymmetric to account for the measurements.

In all six panels of Figure 1, the turbulence has a given configuration. We have redone each of these six simulations for ten different realizations of the turbulence, while leaving $\mathscr{P}(k), B_{\text {rms }}$ and $l_{\mathrm{c}}$ unchanged. We have found that the simulated emissions almost do not vary for small $l_{\mathrm{c}}$. In 

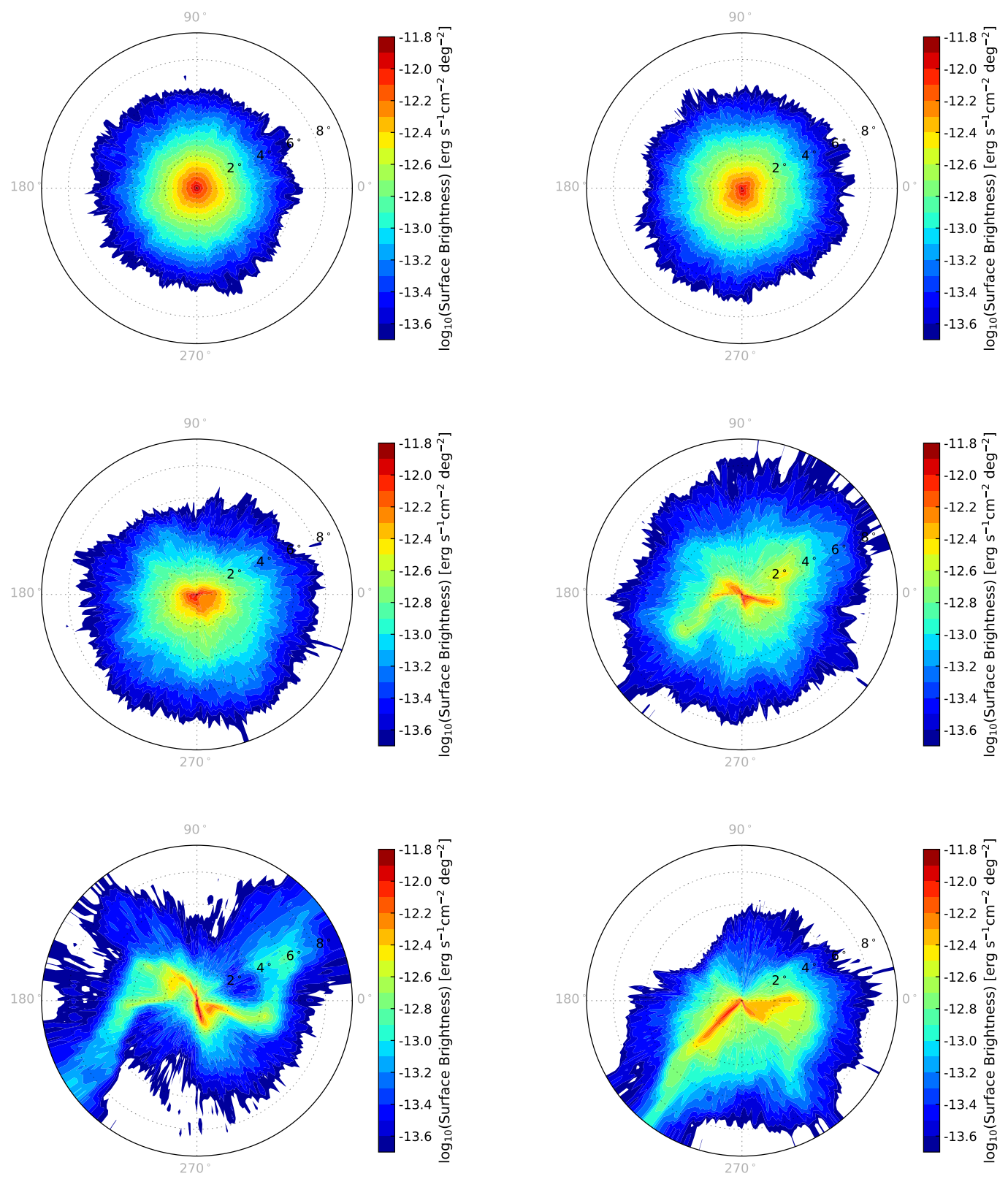

Figure 1: Plots of the simulated gamma-ray surface brightness around Geminga. The pulsar is located at the centre of each plot. Electrons are propagated in given realizations of 3D isotropic Kolmogorov turbulence, with $B_{\mathrm{rms}}=3 \mu \mathrm{G}$ and $l_{\mathrm{c}}=0.25 \mathrm{pc}$ (top left panel), $1 \mathrm{pc}$ (top right), $5 \mathrm{pc}$ (middle left), $10 \mathrm{pc}$ (middle right), $20 \mathrm{pc}$ (bottom left), or $40 \mathrm{pc}$ (bottom right). 
contrast, the shapes of the filaments at large $l_{\mathrm{c}}$ strongly vary from one realization to another. This is not surprizing, given that the geometry of magnetic field lines around the source is completely different from one realization to another. For small values of $l_{\mathrm{c}}$, magnetic field lines are too tangled on the scale of the gamma-ray emission for any substantial difference to be visible in the emission.
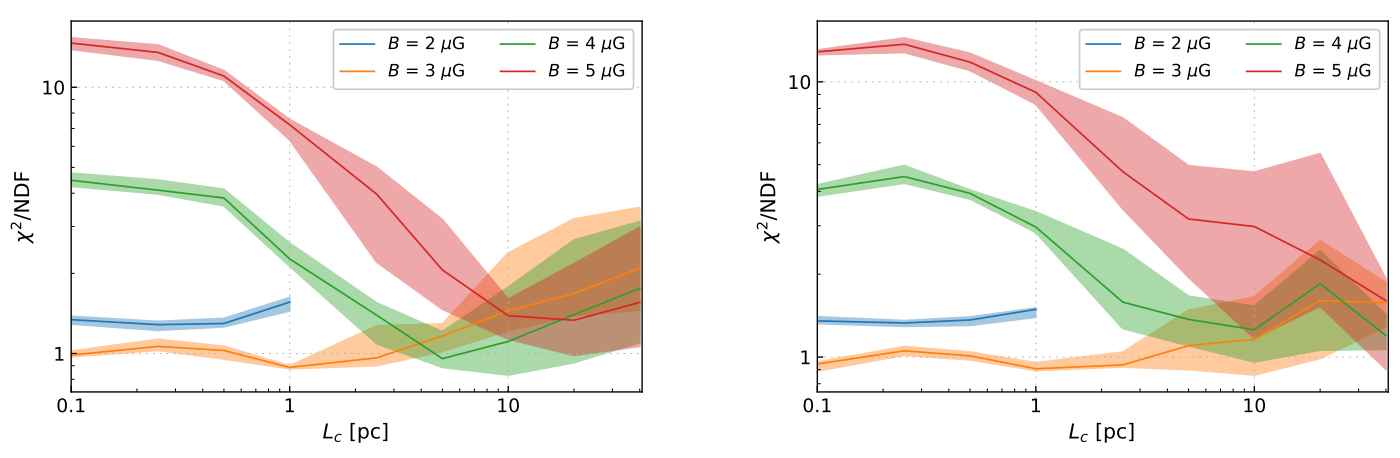

Figure 2: $\chi^{2} /$ ndf versus $l_{\mathrm{c}}$ for our fits to HAWC measurements [3], for Kolmogorov (left panel) and Kraichnan (right) turbulence. Each colour band corresponds to a different value of $B_{\text {rms }}$ - see the values in the keys. The thick solid lines represent the median $\chi^{2} /$ ndf. The widths of the bands provide an estimate the fluctuations from one realization of the turbulence to another - see the text for more details.

For each set of parameters and for each realization, we integrate the gamma-ray surface brightness over all azimuthal angles, plot it versus the angular distance to the pulsar, and fit it to HAWC measurements from [3]. Our calculations of the $\chi^{2} /$ ndf of these fits versus $l_{c}$ are presented in Figure 2 for Kolmogorov (left panel) and Kraichnan (right panel) turbulence. Each line colour corresponds to a different value of $B_{\mathrm{rms}}$, ranging from 2 to $5 \mu \mathrm{G}$. See the keys for the colour code. The widths of the bands quantify the fluctuations from one realization of the turbulence to another. More precisely, the shaded regions correspond to the intervals between the 18th and 82nd percentiles of all realizations. Their widths increase with $l_{\mathrm{c}}$ for the reason discussed above. The thick lines inside each band provide the median values of $\chi^{2} /$ ndf. For both models of the turbulence, the best fits are obtained for $B_{\mathrm{rms}} \simeq 3 \mu \mathrm{G}$ and $l_{\mathrm{c}} \simeq 1 \mathrm{pc}$, where $\chi^{2} /$ ndf $<1$. The value of $l_{\mathrm{c}}$ at which the best fit is obtained for each $B_{\mathrm{rms}}$ increases with the value of $B_{\mathrm{rms}}$. Too weak $\left(B_{\mathrm{rms}} \lesssim 2 \mu \mathrm{G}\right)$ or too strong $\left(B_{\mathrm{rms}} \gtrsim 5 \mu \mathrm{G}\right)$ magnetic fields do not fit HAWC measurements. The fact that the measurements are integrated over all azimuthal angles does not allow us to take into account the constraint from the symmetry of the emission in this fit. In reality, the regions at $l_{\mathrm{c}} \gtrsim 10 \mathrm{pc}$ in these plots are excluded for this reason. We also note that there is no substantial difference between the two panels of Figure 2, which shows that one cannot distinguish between the two power-spectra $\mathscr{P}(k)$ with the current measurements. However, future analyses of the dependence of the emission on gamma-ray energy will be able to provide stronger constraints on $\mathscr{P}(k)$.

In Figure 3, we plot with an orange line the gamma-ray surface brightness - integrated over the azimuthal angle - versus the angular distance to the pulsar for our best fit $\left(l_{\mathrm{c}}=1 \mathrm{pc}, B_{\mathrm{rms}}=\right.$ $3 \mu \mathrm{G}$ ) with Kolmogorov turbulence. HAWC measurements [3] are plotted with the black dots, and the black line corresponds to the fit from the Collaboration [3]. 


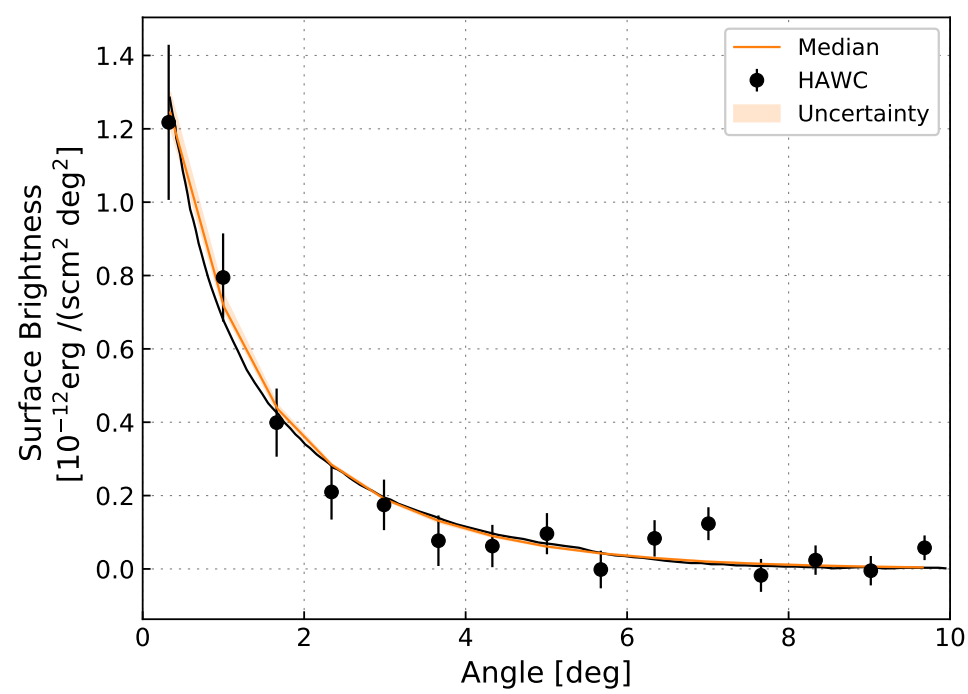

Figure 3: Gamma-ray surface brightness integrated over the azimuthal angle versus the angular distance to the pulsar for our best fit (orange line) to HAWC measurements [3] (black dots): Kolmogorov turbulence with $l_{\mathrm{c}}=1 \mathrm{pc}$ and $B_{\mathrm{rms}}=3 \mu \mathrm{G}$. The black line is the fit from the HAWC Collaboration [3].

\section{Discussion}

The apparent symmetry of the emission detected by HAWC hints at a predominantly turbulent field in the region around Geminga: the amplitude of the large-scale magnetic field in this region should be smaller than that of the turbulent field. This is the reason why we have not added any regular magnetic field in our calculations above. In the presence of a strong regular field, the distribution of electrons and thence the resulting gamma-ray emission would be elongated along the direction of this field. Such a scenario would be compatible with HAWC observations only if the field points in our direction, so that the gamma-ray emission does not appear asymmetric when viewed from the Earth. Although unlikely, this is an interesting possibility because it may help reconcile the small extent of the gamma-ray emission detected by HAWC with a larger CR diffusion coefficient along the line-of-sight, matching the "Galactic average" deduced from the boron-tocarbon ratio. If the regular magnetic field $\mathbf{B}_{0}$ points towards us, the size of the gamma-ray emission would look relatively small because it would be controlled by perpendicular diffusion. In contrast, electrons would diffuse much faster along $\mathbf{B}_{0}$ due to parallel diffusion. The resulting asymmetry of the electron distribution around Geminga would be undetectable by an observer at Earth as long as the angle between $\mathbf{B}_{0}$ and the line-of-sight remains smaller than $\theta \approx \arctan \left(\sqrt{D_{\perp} / D_{\|}}\right)$, where $D_{\perp}$ and $D_{\|}$are the perpendicular and parallel diffusion coefficients. Turbulence levels $B_{\mathrm{rms}} /\left|\mathbf{B}_{0}\right| \leq 0.5$ are needed for $D_{\|}$to reach "Galactic average" values with Kolmogorov turbulence, as can be seen in Figure 3 of Reference [10]. This corresponds to $D_{\|} / D_{\perp} \geq 200$, and thence $\theta \leq 4^{\circ}$. Such a nearperfect alignment of $\mathbf{B}_{0}$ with the line-of-sight, and the presence of such a strong regular field in this region, are quite unlikely. Indeed, $B_{\mathrm{rms}} /\left|\mathbf{B}_{0}\right|$ is usually greater than 1 in the Galactic disc, and 
the regular field should follow spiral arms, which is in contradiction with the fact that the direction to Geminga is not aligned with that of the Orion Spur. Therefore, very-high-energy electrons from Geminga are unlikely to reach us. However, a nearby undiscovered old pulsar could explain the high-energy part of the CR electron spectrum measured at Earth [11].

Finally, we note that the electrons around Geminga might be probing turbulence generated by CRs because $\mathscr{D}_{100}$ is not far from the Bohm value. CR self-confinement around their sources has been studied in a number of papers, see for instance [12, 13, 14, 15]. Reference [16] has recently suggested that it may be the reason for the low value of $\mathscr{D}_{100}$ around Geminga. Such a scenario is possible and interesting, but our study shows that the current HAWC measurements can still be fitted with Kolmogorov or Kraichnan turbulence without requiring such an explanation at the present time. Also, radio observations suggest that the coherence length of the turbulence in the spiral arms of our Galaxy is equal to only a few parsecs, which is very close to our best fit value. See for instance References [17, 18] where the outer scale is found to be $\leq 20 \mathrm{pc}$ (the outer scale is equal to $5 \times l_{\mathrm{c}}$ for Kolmogorov turbulence).

\section{Conclusions and perspectives}

We have demonstrated that the extended gamma-ray emission detected by HAWC around Geminga is compatible with expectations for electrons propagating and cooling in Kolmogorov or Kraichnan turbulence with reasonable strengths and coherence lengths, even if the diffusion coefficient of these electrons is substantially smaller than that usually inferred from the boron-tocarbon ratio for the "Galactic average". Our best fit is obtained for a turbulent magnetic field with root-mean-square strength of $3 \mu \mathrm{G}$ and coherence length of $\simeq 1 \mathrm{pc}$. Magnetic field strengths $<5 \mu \mathrm{G}$ are favoured. Thanks to the lack of strong asymmetries in the observed gamma-ray emission, we can exclude coherence lengths $\gtrsim 10 \mathrm{pc}$ in this $\simeq 25 \mathrm{pc}$-radius region around Geminga. The powerspectrum of the turbulence is not well constrained at the present time, but we expect that one will be able to place more stringent constraints on $\mathscr{P}(k)$ by studying the energy-dependence of the morphology of the emission.

\section{Acknowledgments}

We thank Jim Hinton and the HAWC Collaboration for useful discussions. The research of GG was supported by a Grant from the GIF, the German-Israeli Foundation for Scientific Research and Development.

\section{References}

[1] G. Giacinti, M. Kachelriess, D. V. Semikoz, Filamentary Diffusion of Cosmic Rays on Small Scales, Phys. Rev. Lett. 108 (2012) 261101 [arXiv: 1204 .1271].

[2] G. Giacinti, M. Kachelriess, D. V. Semikoz, Anisotropic Cosmic Ray Diffusion and its Implications for Gamma-Ray Astronomy, Phys. Rev. D 88 (2013) 023010 [arXiv:1306.3209].

[3] A. U. Abeysekara et al. [HAWC Collaboration], Extended gamma-ray sources around pulsars constrain the origin of the positron flux at Earth, Science 358 (2017) 911 [arXiv:1711.06223]. 
[4] R. López-Coto, G. Giacinti, Constraining the properties of the magnetic turbulence in the Geminga region using HAWC $\gamma$-ray data, MNRAS 479 (2018) 4526 [arXiv : 1712.04373 ].

[5] G. Giacinti, J. G. Kirk, Acceleration of X-ray Emitting Electrons in the Crab Nebula, ApJ 863 (2018) 18 [arXiv: 1804.05056$]$.

[6] R. López-Coto, J. Hahn, S. BenZvi, B. Dingus, J. Hinton, M. U. Nisa, R. D. Parsons, F. S. Greus, $\mathrm{H}$. Zhang, H. Zhou, Effect of the diffusion parameters on the observed $\gamma$-ray spectrum of sources and their contribution to the local all-electron spectrum: The EDGE code, Astropart. Phys. 102 (2018) 1 [arXiv:1709.07653].

[7] R. Moderksi, M. Sikora, P. S. Coppi, F. A. Aharonian, Klein-Nishina effects in the spectra of non-thermal sources immersed in external radiation fields, MNRAS 363 (2005) 954, Erratum: MNRAS 364 (2005) 1488 [astro-ph/ 0504388 ].

[8] G. Giacinti, M. Kachelriess, D. V. Semikoz, G. Sigl, Cosmic Ray Anisotropy as Signature for the Transition from Galactic to Extragalactic Cosmic Rays, JCAP 1207 (2012) 031 [arXiv:1112.5599].

[9] J. Hahn, GAMERA - a new modeling package for non-thermal spectral modeling, in proceedings of the 34th International Cosmic Ray Conference, PoS ( ICRC2015) 917 (2016).

[10] G. Giacinti, M. Kachelriess, D. V. Semikoz, Reconciling cosmic ray diffusion with Galactic magnetic field models, JCAP 1807 (2018) 051 [arXiv: 1710 . 08205].

[11] R. López-Coto, R. D. Parsons, J. A. Hinton, G. Giacinti, Undiscovered Pulsar in the Local Bubble as an Explanation of the Local High Energy Cosmic Ray All-Electron Spectrum, Phys. Rev. Lett. 121 (2018) 251106 [arXiv:1811.04123].

[12] V. S. Ptuskin, V. N. Zirakashvili, A. A. Plesser, Non-linear diffusion of cosmic rays, Adv. Space Res. 42 (2008) 486.

[13] M. A. Malkov, P. H. Diamond, R. Z. Sagdeev, F. A. Aharonian, I. V. Moskalenko, Analytic Solution for Self-regulated Collective Escape of Cosmic Rays from their Acceleration Sites, ApJ 768 (2013) 73 [arXiv:1207.4728].

[14] M. D'Angelo, P. Blasi, E. Amato, Grammage of cosmic rays around Galactic supernova remnants, Phys. Rev. D 94 (2016) 083003 [arXiv: 1512.05000 ].

[15] L. Nava, S. Gabici, A. Marcowith, G. Morlino, V. S. Ptuskin, Non-linear diffusion of cosmic rays escaping from supernova remnants - I. The effect of neutrals, MNRAS 461 (2016) 3552 [arXiv:1606.06902].

[16] C. Evoli, T. Linden, G. Morlino, Self-generated cosmic-ray confinement in TeV halos: Implications for TeV $\gamma$-ray emission and the positron excess, Phys. Rev. D 98 (2018) 063017 [arXiv: 1807.09263 ].

[17] M. Haverkorn, J. C. Brown, B. M. Gaensler, N. M. McClure-Griffiths, The outer scale of turbulence in the magneto-ionized Galactic interstellar medium, ApJ 680 (2008) 362 [arXiv: 0802 . 2740 ].

[18] M. Iacobelli et al., Studying Galactic interstellar turbulence through fluctuations in synchrotron emission: First LOFAR Galactic foreground detection, Astron. Astrophys. 558 (2013) A72 [arXiv: 1308.2804$]$. 